\title{
Nanostructured Materials: Bioengineering Platforms for Sensing Nucleic Acids
}

ME Ali and MM Rahman, Nanotechnology and Catalysis Research Centre, Institute of Graduate Studies, University of Malaya, Kuala Lumpur, Malaysia

TS Dhahi, Basra University, Basra, Iraq

M Kashif, University of Malaysia Sarawak, Kota Samarahan, Sarawak, Malaysia

MS Sarkar, Universiti Malaysia Pahang, Gambang, Kuantan, Malaysia

WJ Basirun and SBA Hamid, Nanotechnology and Catalysis Research Centre, Institute of Graduate Studies, University of Malaya, Kuala Lumpur, Malaysia

SK Bhargava, College of Science, Engineering and Health, RMIT University, Melbourne, VIC, Australia

2016 Elsevier Inc. All rights reserved.

\begin{tabular}{llr}
\hline $\mathbf{1}$ & Introduction & 1 \\
$\mathbf{2}$ & Nano-Engineering Platforms for DNA Sensors & 2 \\
2.1 & Nanostructured Gold & 2 \\
2.2 & Nanostructured Silica & 5 \\
2.3 & Semiconductor Nanogap Electrodes & 6 \\
2.4 & Carbon Nanotubes & 6 \\
2.5 & Graphene & 10 \\
2.6 & Nanofluidics & 11 \\
2.7 & Dendrimers & 12 \\
2.8 & Nanowires & 13 \\
2.9 & Quantum Dots & 13 \\
2.10 & Other Nanomaterials & 16 \\
$\mathbf{3}$ & Nanobiofusion Chemistry & 19 \\
3.1 & Biofusion Strategies & 19 \\
3.1 .1 & Physisorption and chemisorption & 19 \\
3.1 .2 & Affinity coupling & 20 \\
3.1 .3 & Covalent attachment & 21 \\
$\mathbf{4}$ & Conclusion and Future Potential & 23 \\
Acknowledgments $\quad$ & 24 \\
References $\quad$ Rurther Reading & & 24 \\
Fur & 25 \\
\hline
\end{tabular}

\section{Introduction}

Current DNA detection techniques are based on polymerase chain reaction assays or fluorophore-labeled sensing systems that suffer from inaccuracy due to various rates of fluorophore photobleaching. Moreover, these methods involve complicated algorithms, expensive fluorophores, and instrumentation for fluorescence readout, which have limited their use in routine analysis (Tansil and Gao, 2006). Thus, there is a need for inexpensive, fast, and reliable molecular techniques for the detection of even a minute amount of DNA (Li et al., 2003). Consequently, nanostructured materials and detection devices for ultrasensitive DNA detection have evolved. In fact, the biosensor applications of nanomaterials are very wide, ranging from nanometric devices in computer chips to the coatings of giant industrial components. Nanolayers can show superior magneto-resistance properties, which have made them suitable for use in the head of a computer hard disk.

Nanostructured materials with unique optical and electrochemical properties greatly facilitate biorecognition of specific DNA targets that often reflect a particular disease or species fingerprints and thus enormously help in point-of-care biodiagnostics (Tansil and Gao, 2006). Higher stability, polymorphic flexibility, and stringent conserved regions of DNA molecules create the opportunity for the identification of specific target sequences with fingerprints for disease diagnostics, paternity testing, and other forensic and biotechnological applications. Definitely, these require tailor-made engineered nanomaterials with cutting-edge useful and attractive properties. In fact, nanoplatforms have built various DNA reorganization tools combining spectroscopic, electrochemistry, magnetic, and other analytical tools (Josephson et al., 2001; Rosi and Mirkin, 2005). In nanoscale sensing, the synergistic effects of functionalized nanomaterials and immobilized DNA probes provide effective recognition of specific DNA targets (Kim et al., 2008).

The fusion of nanomaterials with specific probe DNA or single-stranded DNA (ssDNA) is the key point for development of DNA biosensors. The DNA hybridization detection depends on the physio-chemical properties of the materials or their hybrids 